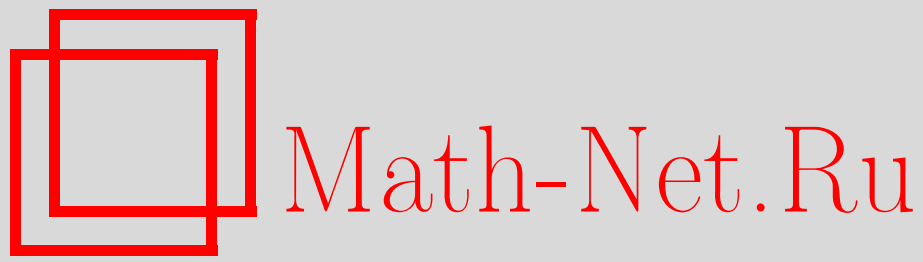

Ю. И. Любарский, В. А. Марченко, Прямая и обратная задачи многоканального рассеяния, Функи. анализ и его прил., 2007, том 41, выпуск 2, 58-77

DOI: https://doi.org/10.4213/faa2861

Использование Общероссийского математического портала MathNet.Ru подразумевает, что вы прочитали и согласны с пользовательским соглашением http://www . mathnet.ru/rus/agreement

Параметры загрузки:

IP: 3.81 .55 .215

26 апреля 2023 г., 12:24:03

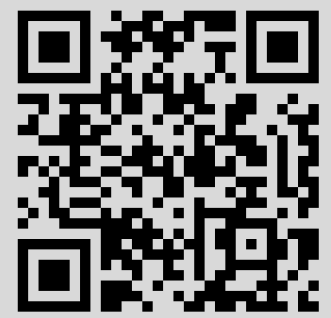




\title{
Прямая и обратная задачи многоканального рассеяния
}

\author{
(С) 2007. Ю. И. ЛЮБАРСКИЙ, В. А. МАРченкО
}

Посвящается памяти М. Г. Крейна

\section{§1. Введение}

Рассмотрим систему частиц $\alpha, \beta, \ldots$ с массами $m(\alpha), m(\beta), \ldots$, взаимодействующих друг с другом, а также, возможно, с внешним полем. Мы изучаем малые колебания таких систем около устойчивого равновесия, предполагая, что потенциальная энергия имеет вид

$$
U=\frac{1}{4} \sum_{\alpha \in \mathscr{A}} \sum_{\beta \in \mathscr{A}} k(\alpha, \beta)|\mathbf{r}(\alpha)-\mathbf{r}(\beta)|^{2}+\frac{1}{2} \sum_{\alpha \in \mathscr{A}} k(\alpha, \alpha)|\mathbf{r}(\alpha)|^{2}
$$

здесь $\mathscr{A}$ обозначает множество всех частиц, $\mathbf{r}(\alpha)$ - отклонение частицы $\alpha$ от положения равновесия, $k(\alpha, \beta)=k(\beta, \alpha) \geqslant 0$ - коэффициент взаимодействия между частицами $\alpha$ и $\beta$, а $k(\alpha, \alpha) \geqslant 0$ - коэффициент взаимодействия частицы $\alpha$ с внешним полем. Функция Лагранжа этой системы имеет вид

$$
\mathscr{L}=\frac{1}{2} \sum_{\alpha \in \mathscr{A}} m(\alpha)|\dot{\mathbf{r}}(\alpha)|^{2}-\frac{1}{4} \sum_{\alpha \in \mathscr{A}} \sum_{\beta \in \mathscr{A}} k(\alpha, \beta)|\mathbf{r}(\alpha)-\mathbf{r}(\beta)|^{2}-\frac{1}{2} \sum_{\alpha \in \mathscr{A}} k(\alpha, \alpha)|\mathbf{r}(\alpha)|^{2} .
$$

Соответственно уравнения движения системы

$$
m(\alpha) \ddot{\mathbf{r}}(\alpha)+\sum_{\beta \in \mathscr{A}} k(\alpha, \beta)(\mathbf{r}(\alpha)-\mathbf{r}(\beta))+k(\alpha, \alpha) \mathbf{r}(\alpha)=0, \quad \alpha \in \mathscr{A},
$$

распадаются на три системы скалярных уравнений. Мы получаем систему

$$
m(\alpha) \ddot{x}(\alpha)+\sum_{\beta \in \mathscr{A}} k(\alpha, \beta)(x(\alpha)-x(\beta))+k(\alpha, \alpha) x(\alpha)=0, \quad \alpha \in \mathscr{A},
$$

и аналогичные системы относительно $y$ - и $z$-координат. В дальнейшем мы ограничиваемся рассмотрением системы (1), описывающей колебания вдоль оси $x$.

Свободными колебаниями называются ограниченные решения системы (1) вида

$$
x(\alpha)=\operatorname{Re}\left(\frac{\xi(\alpha)}{\sqrt{m(\alpha)}} e^{-i \omega t}\right), \quad \omega>0 .
$$

Подставляя эти выражения в (1), мы получим

$$
(L-\lambda I) \xi=0
$$

здесь $\xi=\xi(\alpha)(\alpha \in \mathscr{A}), \lambda=\omega^{2}$ и $L$ - оператор с матрицей $(L(\alpha, \beta))_{\alpha, \beta \in \mathscr{A}}$, где

$$
L(\alpha, \alpha)=\frac{1}{m(\alpha)} \sum_{\beta \in \mathscr{A}} k(\alpha, \beta), \quad L(\alpha, \beta)=-\frac{k(\alpha, \beta)}{\sqrt{m(\alpha) m(\beta)}} .
$$


Частицы $\alpha$ и $\beta$ взаимодействуют друг с другом, если $L(\alpha, \beta) \neq 0$.

Определение. Последовательность частиц $\sigma=\left\{\alpha_{p}\right\}_{p=0}^{\infty}$ называется каналом, если при $p>0$ частица $\alpha_{p}$ взаимодействует только с $\alpha_{p-1}$ и $\alpha_{p+1}$ (и, возможно, с внешним полем), а $\alpha_{0}$ взаимодействует с $\alpha_{1}$ и еще по меньшей мере с двумя частицами, не принадлежащими последовательности $\left\{\alpha_{p}\right\}_{p=0}^{\infty}$.

Введем следующие обозначения:

- множество всех каналов обозначим через $\mathscr{C}$, собственно каналы будут обозначаться через $\sigma, \nu, \gamma$ и т. д.;

- через $\sigma(0), \sigma(1), \sigma(2), \ldots$ обозначим частицы, принадлежащие каналу $\sigma \in$ $\mathscr{C}$; частица $\sigma(0)$ называется концом канала $\sigma$;

- при $k=1,2, \ldots$ положим

$$
-\mathfrak{b}_{\sigma}(k-1)=L(\sigma(k-1), \sigma(k))=L(\sigma(k), \sigma(k-1)), \quad \mathfrak{a}_{\sigma}(k)=L(\sigma(k), \sigma(k)) ;
$$

- $\Gamma:=\{\sigma(0)\}_{\sigma \in \mathscr{C}}, \mathscr{A}_{0}:=\bigcup_{\sigma \in \mathscr{C}} \bigcup_{k=1}^{\infty}\{\sigma(k)\}, \mathscr{A}_{1}:=\mathscr{A} \backslash \mathscr{A}_{0} ;$

- для счетного множества $X$ через $\mathscr{M}(X)$ обозначим векторное пространство всех функций на $X$, а через $l^{2}(X) \subset \mathscr{M}(X)-$ гильбертово пространство функций, суммируемых с квадратом на $X$, с естественным скалярным произведением: $\langle\xi, \eta\rangle=\sum_{x \in X} \xi(x) \overline{\eta(x)}$.

Все пары $(\alpha, \beta) \in \mathscr{A}_{1} \times \mathscr{A}_{0}$, для которых $L(\alpha, \beta) \neq 0$, имеют вид $(\sigma(0), \sigma(1))$, $\sigma \in \mathscr{C}$. Кроме того, если $\alpha=\sigma(k) \in \mathscr{A}_{0}$, то $L(\alpha, \beta) \neq 0$, только когда $\beta \in$ $\{\sigma(k-1), \sigma(k), \sigma(k+1)\}$. Поэтому для того, чтобы функция $\xi \in \mathscr{M}(\mathscr{A})$ являлась решением уравнения (3), необходимо и достаточно, чтобы

$$
\begin{aligned}
-\mathfrak{b}_{\sigma}(k-1) \xi(\sigma(k-1))+\mathfrak{a}_{\sigma}(k) \xi(\sigma(k)) & -\mathfrak{b}_{\sigma}(k) \xi(\sigma(k+1)) \\
& =\lambda \xi(\sigma(k)), \quad \sigma \in \mathscr{C}, k=1,2, \ldots,
\end{aligned}
$$

и

$$
\lambda \xi(\alpha)-\sum_{\beta \in \mathscr{A}_{1}} L(\alpha, \beta) \xi(\beta)=\sum_{\beta \in \mathscr{A}_{0}} L(\alpha, \beta) \xi(\beta)=\sum_{\nu \in \mathscr{C}} L(\alpha, \nu(1)) \xi(\nu(1)), \quad \alpha \in \mathscr{A}_{1} .
$$

Матрица $L_{1}=(L(\alpha, \beta))_{\alpha, \beta \in \mathscr{A}_{1}}$ является подматрицей матрицы $L$ и поэтому строго положительна. Обозначим ее собственные значения и соответствующие им ортонормированные собственные векторы через $0<\lambda_{1} \leqslant \lambda_{2} \leqslant \ldots \leqslant \lambda_{M}$ и $p_{1}, \ldots, p_{M} \in l^{2}\left(\mathscr{A}_{1}\right)$, где $M:=\# \mathscr{A}_{1}=\operatorname{dim} l^{2}\left(\mathscr{A}_{1}\right)$. Без уменьшения общности мы можем считать $p_{j}$ вещественными. В дальнейшем мы не будем различать матрицы и отвечающие им операторы.

При $\lambda \notin\left\{\lambda_{l}\right\}_{l=1}^{M}$ оператор $L_{1}-\lambda I$ обратим; поэтому соотношение $(7)$ может быть записано в виде

$$
\begin{aligned}
\xi(\alpha) & =-\sum_{\beta \in \mathscr{A}_{1}} r(\alpha, \beta ; \lambda) \sum_{\nu \in \mathscr{C}} L(\beta, \nu(1)) \xi(\nu(1)) \\
& =\sum_{\nu \in \mathscr{C}} r(\alpha, \nu(0) ; \lambda) \mathfrak{b}_{\nu}(0) \xi(\nu(1)), \quad \alpha \in \mathscr{A}_{1},
\end{aligned}
$$

где $r(\alpha, \beta ; \lambda)$ - матричные элементы резольвенты $\mathscr{R}=\left(L_{1}-\lambda I\right)^{-1}$ матрицы $L_{1}$ :

$$
\mathscr{R}(\lambda)=(r(\alpha, \beta ; \lambda))_{\alpha, \beta \in \mathscr{A}_{1}}, \quad r(\alpha, \beta ; \lambda)=\sum_{l=1}^{M} \frac{p_{l}(\alpha) p_{l}(\beta)}{\lambda_{l}-\lambda} .
$$


При $\alpha=\sigma(0), \sigma \in \mathscr{C}$, получаем

$$
\xi(\sigma(0))=\sum_{\nu \in \mathscr{C}} r(\sigma(0), \nu(0) ; \lambda) \mathfrak{b}_{\nu}(0) \xi(\nu(1)), \quad \sigma \in \mathscr{C} .
$$

Это соотношение связывает значения решения $\xi$ на $\mathscr{A}_{0}$ и на $\Gamma \subset \mathscr{A}_{1}$. Мы называем его граничным условием.

Теперь нетрудно доказать следующее простое, но важное утверждение.

Теорема 1. Пусть $\lambda \neq \lambda_{l}, l=1, \ldots, M$. Для того чтобъ функиия $\xi(\alpha) н а$ $\mathscr{A}_{0} \cup \Gamma$ могла быть продолжена на все $\mathscr{A}$ до функиии $\xi \in \mathscr{M}(\mathscr{A})$, удовлетворяющей уравнению (3), необходимо и достаточно, чтобы $\xi(\alpha)$ удовлетворяла соотношениям (6) и граничному условию (10). В этом случае продолэсение единственно и определяется по формуле (8).

Мы предполагаем, что каналы однородны на бесконечности, т. е. для всех $\sigma \in \mathscr{C}$ существуют независимые от $\sigma$ пределы

$\lim _{n \rightarrow \infty} k(\sigma(n), \sigma(n+1))=k>0, \lim _{n \rightarrow \infty} k(\sigma(n), \sigma(n))=0, \lim _{n \rightarrow \infty} m(\sigma(n))=m>0$, и, более того,

$$
\sum_{n>0} n(|k(\sigma(n), \sigma(n+1))-k|+|k(\sigma(n), \sigma(n))|+|m(\sigma(n))-m|)<\infty .
$$

Тогда из соотношений (4) и (5) следует, что

$$
\mathfrak{a}_{\sigma}(n) \rightarrow 2 \frac{k}{m} \quad \text { и } \quad \mathfrak{b}_{\sigma}(n) \rightarrow \frac{k}{m} \quad \text { при } n \rightarrow \infty, \quad \sigma \in \mathscr{C} .
$$

Отношение $k / m$ имеет размерность сек ${ }^{-2}$; поэтому, не уменьшая общности, можно считать, что $k / m=1$.

Таким образом, мы изучаем системы частиц, обладающие следующими свойствами:

А) они состоят из конечного числа каналов и, кроме того, конечного числа частии, не принадлежащих этим каналам;

В) $\sum_{1}^{\infty} n\left\{\left|\mathfrak{b}_{\sigma}(n)-1\right|+\left|\mathfrak{a}_{\sigma}(n)-2\right|\right\}<\infty$ для всех $\sigma \in \mathscr{C}$.

При $n \rightarrow \infty$ (т. е. на однородных частях каналов) колебания таких систем могут быть описаны простыми асимптотическими соотношениями. Эти соотношения включают параметры, называемые данными рассеяния, значения которых определяются физическими свойствами системы.

Прямая задача теории рассеяния заключается в нахождении данных рассеяния по заданным физическим характеристикам системы. При решении обратной задачи требуется определить, какие физические характеристики системы могут быть определены по данным рассеяния, и, главное, найти численные значения этих характеристик.

В этой работе мы рассматриваем задачу рассеяния для систем, удовлетворяющих условиям А) и В). Мы решаем прямую задачу, а также восстанавливаем характеристики каналов (т. е. значения $L(\alpha, \beta)$ при $\alpha, \beta \in \mathscr{A}_{0}$ ) по данным рассеяния. В следующей работе мы укажем условия, при которых по данным рассеяния возможно также найти $L(\alpha, \beta)$ для $\alpha, \beta \in \mathscr{A}_{1}$ и для $\alpha \in \mathscr{A}_{0}, \beta \in \mathscr{A}_{1}$ и, тем самым, восстановить всю матрицу $L$.

Аналогичные вопросы, относящиеся к рассеянию на квантовых графах, интенсивно изучались в последнее время, см., например, [2], [4]-[8], а также ссылки, приведенные в этих работах. 


\section{§2. Свойства решений конечно-разностных уравнений}

В этом параграфе мы приводим известные (см., например, [9]) свойства решений конечно-разностного уравнения

$$
-\mathfrak{b}(k-1) x(k-1)+\mathfrak{a}(k) x(k)-\mathfrak{b}(k) x(k+1)=\lambda x(k), \quad k=1,2, \ldots,
$$

с вещественными коэффициентами $\mathfrak{a}(k), \mathfrak{b}(k)$.

Вронскианом функций $x, y$, определенных на множестве неотрицательных целых чисел, называется функция

$$
\{x, y\}(k)=x(k) \overline{y(k+1)}-x(k+1) \overline{y(k)}, \quad k=0,1, \ldots
$$

1. Пусть $x$ является решением уравнения (12). Тогда при всех $N>0$

$$
\mathfrak{b}(N)\{x, x\}(N)-\mathfrak{b}(0)\{x, x\}(0)=(\lambda-\bar{\lambda}) \sum_{k=1}^{N}|x(k)|^{2} .
$$

Если, кроме того, $\lambda=\lambda(\theta)$ и $x=x(k, \theta)$ являются дифференцируемыми функциями от параметра $\theta$, то

$$
\mathfrak{b}(N)\{\dot{x}, x\}(N)-\mathfrak{b}(0)\{\dot{x}, x\}(0)=\dot{\lambda} \sum_{k=1}^{N}|x(k)|^{2}+(\lambda-\bar{\lambda}) \sum_{k=1}^{N} \dot{x}(k) \overline{x(k)} ;
$$

здесь и в дальнейшем мы обозначаем точкой дифференцирование по $\theta$.

2. Пусть, кроме того,

$$
\sum_{k=1}^{\infty} k(|\mathfrak{b}(k)-1|+|\mathfrak{a}(k)-2|)<\infty
$$

Положим

$$
\lambda=\lambda(\theta)=2-\left(\theta+\theta^{-1}\right) .
$$

Тогда для всех $\theta \neq 0$, принадлежащих замыканию $\overline{\mathbb{D}}$ единичного круга, уравнения (12) имеют решения вида

$$
e(k, \theta)=c(k) \sum_{m \geqslant k} a(k, m) \theta^{m}, \quad k=0,1, \ldots
$$

Здесь

$$
c(k)=\left(\prod_{p=k}^{\infty} b(p)\right)^{-1}, \quad a(k, k)=1 \quad \text { и } \quad \lim _{k \rightarrow \infty} \sum_{m \geqslant k+1}|a(k, m)|=0 .
$$

Решения $e(k, \theta)$ голоморфны по $\theta$ в $\mathbb{D}$, непрерывны в $\overline{\mathbb{D}}$ и

$$
e(k, \theta)=\theta^{k}(1+o(1)), \quad k \rightarrow \infty,
$$

равномерно относительно $\theta \in \overline{\mathbb{D}}$.

При $k \geqslant 1$ коэффициенты $\mathfrak{a}(k), \mathfrak{b}(k)$ могут быть определены из соотношений

$$
\begin{gathered}
\mathfrak{a}(k)-2=a(k-1, k)-a(k, k+1), \\
\mathfrak{b}(k)^{2}=(\mathfrak{a}(k)-2) a(k, k+1)+a(k, k+2)-a(k-1, k+1)+1 .
\end{gathered}
$$

Решения $e(k, \theta)$ и их представления (15) являются дискретными аналогами классических решений Йоста и операторов преобразования Левина. 
Подставим в $(13) \lambda=\lambda(\theta), x(k)=e(k, \theta)$ и устремим $N$ к бесконечности. С учетом соотношения (17) получаем

$$
\mathfrak{b}(0)\{e, e\}(0)= \begin{cases}\bar{\theta}-\theta, & \text { если }|\theta|=1, \\ (\bar{\theta}-\theta)\left(|\theta|^{-2}-1\right) \sum_{k=1}^{\infty}|e(k, \theta)|^{2}, & \text { если }|\theta|<1 .\end{cases}
$$

При $N \rightarrow \infty$ соотношение (14) с $-1<\theta<1, x(k, \theta)=\bar{x}(k, \theta)=e(k, \theta)$ и $\lambda=\lambda(\theta)$ дает

$$
-\mathfrak{b}(0)\{\dot{e}, e\}(0)=\left(\theta^{-2}-1\right) \sum_{k=1}^{\infty} e(k, \theta)^{2}, \quad-1<\theta<1 .
$$

Нам понадобится также следующее утверждение относительно решений уравнения (3).

Лемма 2.1. Пусть функиия $\xi(\alpha)=\xi(\alpha ; \theta)$ дифференцируема по $\theta$ в окрестности точки $\hat{\theta} \in \mathbb{D}, \operatorname{Im} \lambda(\hat{\theta})=0$, и удовлетворяет уравнению

$$
\lambda(\theta) \xi(\alpha ; \theta)=\sum_{\beta \in \mathscr{A}} L(\alpha, \beta) \xi(\beta ; \theta) .
$$

Пусть, кроме того, функиия $\eta(\alpha)$ удовлетворяет этому же уравнению при $\theta=\hat{\theta}$. Тогда

$$
\left(\hat{\theta}^{-2}-1\right) \sum_{\alpha \in \mathscr{A}_{1}} \xi(\alpha ; \hat{\theta}) \overline{\eta(\alpha)}=\sum_{\sigma \in \mathscr{C}} \mathfrak{b}_{\sigma}(0)\{\dot{\xi}(\sigma(\cdot) ; \hat{\theta}), \eta(\sigma(\cdot))\}(0) .
$$

B частности,

$$
\left(\hat{\theta}^{-2}-1\right) \sum_{\alpha \in \mathscr{A}_{1}}|\xi(\alpha ; \hat{\theta})|^{2}=\sum_{\sigma \in \mathscr{C}} \mathfrak{b}_{\sigma}(0)\{\dot{\xi}(\sigma(\cdot) ; \hat{\theta}), \xi(\sigma(\cdot) ; \hat{\theta})\}(0) .
$$

Доказательство. Продифференцируем уравнения (22) по $\theta$ :

С учетом соотношения

$$
\dot{\lambda}(\theta) \xi(\alpha ; \theta)+\lambda(\theta) \dot{\xi}(\alpha ; \theta)=\sum_{\beta \in \mathscr{A}} L(\alpha, \beta) \dot{\xi}(\beta ; \theta) .
$$

получаем

$$
\lambda(\hat{\theta}) \overline{\eta(\alpha)}=\overline{\lambda(\hat{\theta}) \eta(\alpha)}=\sum_{\beta \in \mathscr{A}} L(\alpha, \beta) \overline{\eta(\beta)}
$$

$$
\dot{\lambda}(\hat{\theta}) \xi(\alpha ; \hat{\theta}) \overline{\eta(\alpha)}=\sum_{\beta \in \mathscr{A}} L(\alpha, \beta)(\dot{\xi}(\beta ; \hat{\theta}) \overline{\eta(\alpha)}-\dot{\xi}(\alpha ; \hat{\theta}) \overline{\eta(\beta)})
$$

$$
\begin{aligned}
\dot{\lambda}(\hat{\theta}) \sum_{\alpha \in \mathscr{A}_{1}} \xi\left(\alpha ; \theta_{l}\right) \overline{\eta(\alpha)}=\sum_{\alpha \in \mathscr{A}_{1}} \sum_{\beta \in \mathscr{A}_{1}} L(\alpha, \beta)(\dot{\xi}(\beta ; \hat{\theta}) \overline{\eta(\alpha)}-\dot{\xi}(\alpha ; \hat{\theta}) \overline{\eta(\beta)}) & \\
& +\sum_{\alpha \in \mathscr{A}_{1}} \sum_{\beta \in \mathscr{A}_{0}} L(\alpha, \beta)(\dot{\xi}(\beta ; \hat{\theta}) \overline{\eta(\alpha)}-\dot{\xi}(\alpha ; \hat{\theta}) \overline{\eta(\beta)}) .
\end{aligned}
$$

Первое слагаемое в правой части равно нулю, потому что его члены антисимметричны по $\alpha$ и $\beta$.

Во втором слагаемом коэффициент $L(\alpha, \beta)$ отличен от нуля, только если $\alpha=\sigma(0), \beta=\sigma(1)$ при некотором $\sigma \in \mathscr{C}$, поскольку это единственные пары взаимодействующих частиц из $\mathscr{A}_{1}$ и $\mathscr{A}_{0}$. 
Так как $\dot{\lambda}(\theta)=\theta^{-2}-1$ и $L(\sigma(0), \sigma(1))=-\mathfrak{b}_{\sigma}(0)$ (см. (5)), отсюда получается соотношение $(23)$.

\section{§3. Матрица рассеяния}

Из условий А) и В) вытекает, что уравнения (6) допускают решения Йоста

$$
e_{\sigma}(k, \theta)=c_{\sigma}(k) \sum_{m \geqslant k} a_{\sigma}(k, m) \theta^{m}, \quad k=0,1,2, \ldots
$$

Свойства этих решений описаны в предыдущем параграфе.

Пусть $\theta \in \mathbb{T} \backslash\{ \pm 1\}$ (как всегда, $\mathbb{T}$ обозначает единичную окружность). Тогда $\lambda(\theta)=\overline{\lambda(\theta)}=\lambda\left(\theta^{-1}\right)=\lambda(\bar{\theta})$ и функции $e_{\sigma}(k, \theta)$ и $e_{\sigma}\left(k, \theta^{-1}\right)=\overline{e_{\sigma}(k, \theta)}$ являются линейно независимыми. Поэтому все решения уравнения (6) имеют вид

$$
\xi(\sigma(k))=m(\sigma) e_{\sigma}(k, \theta)+n(\sigma) e_{\sigma}\left(k, \theta^{-1}\right), \quad k=0,1,2, \ldots
$$

В дальнейшем мы обозначаем функции из $l^{2}(\mathscr{C})$ жирными буквами. В частности, $\mathbf{m}=m(\sigma), \mathbf{n}=n(\sigma), \boldsymbol{\xi}(k)=\xi(\sigma, k)$ и т. д. Рассмотрим диагональные матрицы (операторы в $\left.l^{2}(\mathscr{C})\right)$ :

$$
\begin{aligned}
& E(k, \theta):=\operatorname{diag}\left\{e_{\sigma}(k, \theta)\right\}_{\sigma \in \mathscr{C}}, \quad B(k):=\operatorname{diag}\left\{\mathfrak{b}_{\sigma}(k)\right\}_{\sigma \in \mathscr{C}}, \\
& C(k):=\operatorname{diag}\left\{c_{\sigma}(k)\right\}_{\sigma \in \mathscr{C}}, \quad A(k, m):=\operatorname{diag}\left\{a_{\sigma}(k, m)\right\}_{\sigma \in \mathscr{C}},
\end{aligned}
$$

а также матрицы

$$
\begin{aligned}
R(\theta) & =(r(\sigma(0), \nu(0) ; \lambda(\theta)))_{\sigma, \nu \in \mathscr{C}}: l^{2}(\mathscr{C}) \rightarrow l^{2}(\mathscr{C}), \\
R_{1}(\theta) & =(r(\alpha, \nu(0) ; \lambda(\theta)))_{\alpha \in \mathscr{A}_{1}, \nu \in \mathscr{C}}: l^{2}(\mathscr{C}) \rightarrow l^{2}\left(\mathscr{A}_{1}\right),
\end{aligned}
$$

где функции $r(\alpha, \beta ; \lambda)$ определены соотношениями $(9)$. Операторы $A(k, m)$, $B(k), C(k)$, очевидно, являются самосопряженными.

Представления (24), (25) могут быть теперь записаны в виде

$$
\begin{gathered}
E(k, \theta)=C(k) \sum_{m \geqslant k} A(k, m) \theta^{m}, \quad k=0,1,2, \ldots, \\
\boldsymbol{\xi}(k)=E(k, \theta) \mathbf{m}+E\left(k, \theta^{-1}\right) \mathbf{n} .
\end{gathered}
$$

Соответственно граничное условие (10) принимает вид

$$
E(0, \theta) \mathbf{m}+E\left(0, \theta^{-1}\right) \mathbf{n}=R(\theta) B(0)\left(E(1, \theta) \mathbf{m}+E\left(1, \theta^{-1}\right) \mathbf{n}\right),
$$

или

$$
T(\theta) \mathbf{m}=-T\left(\theta^{-1}\right) \mathbf{n},
$$

где

$$
T(\theta):=E(0, \theta)-R(\theta) B(0) E(1, \theta) .
$$

Матрицы $R(\theta)$ и $T(\theta)$ определены для всех $\theta \in \overline{\mathbb{D}} \backslash \mathscr{O}$, где

$$
\mathscr{O}=\left\{\theta \in \overline{\mathbb{D}}: \lambda_{l}-\lambda(\theta)=0 \quad \text { при некотором } l \in\{1, \ldots, M\}\right\} \cup\{-1,0,1\} .
$$

Очевидно, что $\mathscr{O} \subset \mathbb{R} \cup \mathbb{T}$.

Соотношение (20) принимает вид

$$
B(0)\left\{E(0, \theta) E(1, \theta)^{*}-E(0, \theta)^{*} E(1, \theta)\right\}=(\bar{\theta}-\theta) \Delta(\theta),
$$


где

$$
\Delta(\theta)= \begin{cases}I, & |\theta|=1 \\ \left(|\theta|^{-2}-1\right) \sum_{k=1}^{\infty} E(k, \theta) E(k, \theta)^{*}, & |\theta|<1 .\end{cases}
$$

Из соотношения (17) легко следует, что оператор $\Delta(\theta)$ положительно определен равномерно относительно $\theta \in \overline{\mathbb{D}}$, т.е. при некотором $\Delta>0$ имеет место неравенство

$$
\langle\Delta(\theta) \mathbf{x}, \mathbf{x}\rangle \geqslant \Delta\|\mathbf{x}\|^{2}, \quad \mathbf{x} \in l^{2}(\mathscr{C}), \theta \in \overline{\mathbb{D}}
$$

Лемма 3.1. Для всех $\theta \in \overline{\mathbb{D}} \backslash \mathscr{O} u \mathbf{x} \in l^{2}(\mathscr{C})$ справедливо неравенство

$$
\left|\left\langle E(1, \theta)^{*} B(0) T(\theta) \mathbf{x}, \mathbf{x}\right\rangle\right| \geqslant\left|\operatorname{Im}\left\langle E(1, \theta)^{*} B(0) T(\theta) \mathbf{x}, \mathbf{x}\right\rangle\right| \geqslant \frac{|\bar{\theta}-\theta|}{2} \Delta\|\mathbf{x}\|^{2} .
$$

Доказательство. Имеем

$2 i \operatorname{Im}\left\langle E(1, \theta)^{*} B(0) T(\theta) \mathbf{x}, \mathbf{x}\right\rangle=\left\langle\left[E(1, \theta)^{*} B(0) T(\theta)-T(\theta)^{*} B(0) E(1, \theta)\right] \mathbf{x}, \mathbf{x}\right\rangle$, откуда, согласно (31), следует, что

$$
\begin{array}{r}
E(1, \theta)^{*} B(0) T(\theta)-T(\theta)^{*} B(0) E(1, \theta)=B(0)\left(E(0, \theta) E(1, \theta)^{*}-E(0, \theta)^{*} E(1, \theta)\right) \\
+(B(0) E(1, \theta))^{*}\left(R(\theta)^{*}-R(\theta)\right)(B(0) E(1, \theta)) .
\end{array}
$$

Для доказательства леммы достаточно теперь воспользоваться соотношениями (32), (34), а также тем, что

$$
R(\theta)^{*}-R(\theta)=(\bar{\theta}-\theta)\left(|\theta|^{-2}-1\right)\left(\sum_{l=1}^{M} \frac{p_{l}(\sigma(0)) p_{l}(\nu(0))}{\left|\lambda_{l}-\lambda(\theta)\right|^{2}}\right)=:(\bar{\theta}-\theta) \Delta_{1}(\theta),
$$

и тем, что оператор $\Delta_{1}(\theta)$ неотрицательно определен.

Следствие. Oператор $T(\theta)$ обратим для всех невещественных $\theta \in \overline{\mathbb{D}} \backslash \mathscr{O}$.

Из уравнения (30) вытекает, что при всех $\theta \in \mathbb{T} \backslash \mathscr{O}$

$$
\mathbf{m}=S(\theta) \mathbf{n},
$$

где

$$
S(\theta)=-T(\theta)^{-1} T\left(\theta^{-1}\right)=(s(\sigma, \nu ; \theta))_{\sigma, \nu \in \mathscr{C}} .
$$

Соответственно все решения задачи (3) с $\lambda=\lambda(\theta)$ имеют вид

$$
\boldsymbol{\xi}(k ; \theta)=U(k, \theta) \mathbf{n}, \quad k=0,1, \ldots, \quad \xi(\alpha ; \theta)=R_{1}(\theta) B(0) U(1, \theta) \mathbf{n}, \quad \alpha \in \mathscr{A}_{1},
$$

где $\mathbf{n} \in l^{2}(\mathscr{C})$ - произвольный вектор. Здесь матрица $U(k, \theta)$ определена формулой

$$
U(k, \theta)=E\left(k, \theta^{-1}\right)+E(k, \theta) S(\theta), \quad \theta \in \mathbb{T} \backslash \mathscr{O} .
$$

Из соотношений $(2),(11)$ и (17) вытекает, что при $\theta=e^{i \phi} \in \mathbb{T} \backslash \mathscr{O}$ свободные колебания с частотой $\omega=2 \sin (\phi / 2)$ на канале $\sigma$ имеют следующую асимптотику:

$$
\begin{aligned}
x(\sigma(k), t) \sim \frac{1}{\sqrt{m}}( & |n(\sigma)| \cos (k \phi+\omega t-\arg n(\sigma)) \\
& +|m(\sigma)| \cos (k \phi-\omega t+\arg m(\sigma))), \quad k \rightarrow \infty
\end{aligned}
$$


где $m(\sigma)=\sum_{\nu \in \mathscr{C}} s(\sigma, \nu ; \theta) n(\nu)$. Первое слагаемое в правой части формулы $(39)$ описывает падающую волну, распространяющуюся вдоль каналов из бесконечности со скоростью $v=\omega / \phi$. Взаимодействие этих волн с частицами системы порождает рассеянные волны, они отвечают второму слагаемому. Эти волны устремляются к бесконечности с той же скоростью $v$.

Единственная информация, доступная при наблюдении на бесконечности, относится к связи между падающими и рассеянными волнами, иными словами, к зависимости вектора $\mathbf{m}$ от $\mathbf{n}$. Зависимость между этими векторами дается соотношением (36). Поэтому оператор $S(\theta)$ и его матрица называются оператором рассеяния и матрищей рассеяния соответственно.

Лемма 3.2. При $\theta \in \mathbb{T} \backslash \mathscr{O}$ матрица $S(\theta)$ симметрична и унитарна.

Доказательство. Достаточно доказать, что

$$
\|S(\theta) \mathbf{x}\|^{2}=\|\mathbf{x}\|^{2}, \quad \mathbf{x} \in l^{2}(\mathscr{C}), \theta \in \mathbb{T} \backslash \mathscr{O}, \theta \neq \pm 1 .
$$

Положим $\mathbf{y}=S(\theta) \mathbf{x}$. Из соотношения (37) вытекает, что $T(\theta) \mathbf{y}+T\left(\theta^{-1}\right) \mathbf{x}=0$ и, согласно соотношению (30),

$$
E(0, \theta) \mathbf{y}+E\left(0, \theta^{-1}\right) \mathbf{x}=R(\theta) B(0)\left(E(1, \theta) \mathbf{y}+E\left(1, \theta^{-1}\right) \mathbf{x}\right) .
$$

Оператор $R(\theta)$ самосопряжен. Поэтому из последнего равенства следует, что

$$
\operatorname{Im}\left\langle E(0, \theta) \mathbf{y}+E\left(0, \theta^{-1}\right) \mathbf{x}, B(0)\left(E(1, \theta) \mathbf{y}+E\left(1, \theta^{-1}\right) \mathbf{x}\right)\right\rangle=0,
$$

и с учетом того, что $E(k, \theta)^{*}=E\left(k, \theta^{-1}\right), B(0)^{*}=B(0)$, получаем

$$
\begin{aligned}
\operatorname{Im}\left[\left\langle B(0) E(0, \theta) E(1, \theta)^{*} \mathbf{y}, \mathbf{x}\right\rangle+\langle B(0) E(0, \theta) E(1, \theta) \mathbf{y}, \mathbf{x}\rangle\right. \\
\left.+\langle\mathbf{x}, B(0) E(0, \theta) E(1, \theta) \mathbf{y}\rangle+\left\langle\mathbf{x}, B(0) E(0, \theta) E(1, \theta)^{*} \mathbf{x}\right\rangle\right]=0 .
\end{aligned}
$$

Сумма второго и третьего членов в левой части этого равенства вещественна. Поэтому сумма мнимых частей первого и последнего членов равна нулю:

$$
\begin{aligned}
\langle B(0) & \left.\left\{E(0, \theta) E(1, \theta)^{*}-E(0, \theta)^{*} E(1, \theta)\right\} \mathbf{y}, \mathbf{y}\right\rangle \\
& +\left\langle\mathbf{x}, B(0)\left\{E(0, \theta) E(1, \theta)^{*}-E(0, \theta)^{*} E(1, \theta)\right\} \mathbf{x}\right\rangle=0 .
\end{aligned}
$$

Соотношения (32), (33) позволяют переписать это в виде $(\bar{\theta}-\theta)\{\langle\mathbf{y}, \mathbf{y}\rangle-\langle\mathbf{x}, \mathbf{x}\}\rangle$ $=0$, откуда прямо следует (40).

Кроме того, из соотношения (37) вытекает равенство $S(\theta) S\left(\theta^{-1}\right)=I$, а явное выражение для $S$ дает $\overline{S(\theta)}=S\left(\theta^{-1}\right)$. Поэтому

$$
S^{*}(\theta)=S(\theta)^{-1}=S\left(\theta^{-1}\right)=\overline{S(\theta)}, \quad \theta \in \mathbb{T} \backslash \mathscr{O} .
$$

В частности, $S^{*}(\theta)=\overline{S(\theta)}$, т. е. матрица $S(\theta)$ симметрична.

\section{§4. Аналитическое продолжение}

Равенство (38) определяет решение задачи (3) с $\lambda=\lambda(\theta), \theta \in \mathbb{T} \backslash \mathscr{O}$. В этом параграфе мы продолжаем это решение до решения, мероморфного по $\theta$ во всем круге $\mathbb{D}$. Достаточно построить продолжение матрицы $U(k, \theta)$.

Из соотношений $(31)$ и (32) вытекает, что при $\theta \in \mathbb{T} \backslash \mathscr{O}$

$$
\begin{aligned}
T(\theta) E\left(1, \theta^{-1}\right)-T\left(\theta^{-1}\right) E(1, \theta) & =E(0, \theta) E\left(1, \theta^{-1}\right)-E\left(0, \theta^{-1}\right) E(1, \theta) \\
& =\left(\theta^{-1}-\theta\right) B(0)^{-1}
\end{aligned}
$$

3 Функциональный анализ и его приложения, т. 41, вып. 2 
С учетом (37) имеем

$$
S(\theta)=\left\{-E\left(1, \theta^{-1}\right)+\left(\theta^{-1}-\theta\right) T(\theta)^{-1} B(0)^{-1}\right\} E(1, \theta)^{-1}
$$

и

$$
U(k, \theta)=\left(\theta-\theta^{-1}\right)\left\{P(k, \theta)-E(k, \theta) T(\theta)^{-1}\right\} B(0)^{-1} E(1, \theta)^{-1},
$$

где

$$
P(k, \theta)=\left(\theta^{-1}-\theta\right)^{-1} B(0)\left\{E(k, \theta) E\left(1, \theta^{-1}\right)-E\left(k, \theta^{-1}\right) E(1, \theta)\right\} .
$$

Матрица $P(k, \theta)$ является диагональной, ее элементы $p_{\sigma}(k, \theta)$ удовлетворяют уравнению (6), а также (согласно $(20))$ начальным условиям $p_{\sigma}(0, \theta)=1, p_{\sigma}(1, \theta)$ $=0$.

Таким образом, $P(0, \theta)=I, P(1, \theta)=0$, а при $k \geqslant 2 P(k, \theta)$ является полиномом степени $k-2$ относительно $\lambda$. Следовательно, правая часть соотношения (41) определяет продолжение матрицы $U(k, \theta)$ до функции, мероморфной по $\theta$ в единичном круге $\mathbb{D}$.

Из соотношения $(20)$ вытекает, что $e(1, \theta) \neq 0$ при $\theta \notin \mathbb{R}$. Отсюда, с учетом следствия леммы 3.1, можно заключить, что все полюсы функции $U(k, \theta)$ принадлежат интервалу $(-1,1)$. Из соотношения $(41)$ следует, что каждый отличный от нуля полюс этой функции является полюсом либо для $T(\theta)^{-1}$, либо для $E(1, \theta)^{-1}$. Это множество полюсов не зависит от $k$. Мы обозначим его через $\Theta$.

Равенства (38) определяют теперь решения задачи (3) с $\lambda=\lambda(\theta)$ для всех $\theta \in \overline{\mathbb{D}} \backslash(\Theta \cup \mathscr{O})$. В отличие от случая $\theta \in \mathbb{T}$ эти решения, вообще говоря, не являются ограниченными.

Лемма 4.1. Функиия $U(k, \theta)$ имеет полюс порядка $k$ в нуле, а все полюсъ из $\Theta$ являются простьми.

Доказательство. Перепишем представление (41) в виде

$U(k, \theta)=\left(\theta-\theta^{-1}\right) P(k, \theta) B(0)^{-1} E(1, \theta)^{-1}-\left(\theta-\theta^{-1}\right) E(k, \theta) T(\theta)^{-1} B(0)^{-1} E(1, \theta)^{-1}$.

Функции $U(0, \theta)$ и $U(1, \theta)$ имеют в нуле полюсы порядков 0 и 1 соответственно. Это прямо вытекает из начальных условий для $P(k, \lambda)$, представления $(15)$ решений Йоста, а также явного выражения для $R(\theta)$.

При $k \geqslant 2$ главный вклад в особенность функции $U(k, \theta)$ в нуле вносит первое слагаемое в правой части представления (42). Это следует из того, что $P(k, \theta)$ является полиномом степени $k-2$ относительно $\lambda=2-\theta-\theta^{-1}$. При этом так же, как и в случае $k=0,1$, нужно воспользоваться представлением $(15)$ решений Йоста. Мы опускаем подробное вычисление.

Функции $e_{\sigma}(1, \theta)$ и $\dot{e}_{\sigma}(1, \theta)$ не могут иметь общих корней. Это следует из соотношения $(21)$. Поэтому первое слагаемое в правой части формулы (42) может иметь на $(-1,0) \cup(0,1)$ только простые полюсы.

Для анализа второго слагаемого воспользуемся соотношением (35), в котором $\mathbf{x}=T(\theta)^{-1} B(0)^{-1} E(1, \theta)^{-1} \mathbf{y}$, а $\mathbf{y}-$ произвольный вектор из $l^{2}(\mathscr{C})$. Из неравенства Коши следует, что

$$
\begin{aligned}
\frac{|\bar{\theta}-\theta|}{2} \Delta & \left\|T(\theta)^{-1} B(0)^{-1} E(1, \theta)^{-1} \mathbf{y}\right\|^{2} \\
& \leqslant\left|\left\langle E(1, \theta)^{*} E(1, \theta)^{-1} \mathbf{y}, T(\theta)^{-1} B(0)^{-1} E(1, \theta)^{-1} \mathbf{y}\right\rangle\right| \\
& \leqslant\left\|E(1, \theta)^{*} E(1, \theta)^{-1} \mathbf{y}\right\|\left\|T(\theta)^{-1} B(0)^{-1} E(1, \theta)^{-1} \mathbf{y}\right\|
\end{aligned}
$$


Так как оператор $E(1, \theta)^{*} E(1, \theta)^{-1}$ унитарен, то $\left\|E(1, \theta)^{*} E(1, \theta)^{-1} \mathbf{y}\right\|=\|\mathbf{y}\|$ и, окончательно,

$$
\frac{\left\|T(\theta)^{-1} B(0)^{-1} E(1, \theta)^{-1} \mathbf{y}\right\|}{\|\mathbf{y}\|} \leqslant \frac{2}{|\bar{\theta}-\theta| \Delta}, \quad \mathbf{y} \in l^{2}(\mathscr{C}) .
$$

Поэтому на $(-1,0) \cup(0,1)$ матрица-функция $T(\theta)^{-1} B(0)^{-1} E(1, \theta)^{-1}$ может иметь только простые полюсы.

Лемма 4.2. Матрицъ-функиии $\left(\theta-\theta^{-1}\right) T(\theta)^{-1} u\left(\theta-\theta^{-1}\right) E(1, \theta)^{-1}$ имеют конечное число полюсов в $\mathbb{D}$. Кроме того, они ограничены в достаточно малой окрестности точек \pm 1 в $\overline{\mathbb{D}}$.

Доказательство аналогично доказательству леммы 2.4 .1 в [1], и мы его опускаем. Заметим только, что если точка $\theta_{l} \neq 0$ является полюсом одной из функций $T(\theta)^{-1}$ или $E(1, \theta)^{-1}$, то $\lambda\left(\theta_{l}\right)$ является собственным значением либо всей матрицы $(L(\alpha, \beta))_{\alpha, \beta \in \mathscr{A}}$, либо одной из якобиевых матриц

$$
\left(\begin{array}{cccc}
\mathfrak{a}_{\sigma}(1) & -\mathfrak{b}_{\sigma}(1) & 0 & 0 \\
-\mathfrak{b}_{\sigma}(1) & \mathfrak{a}_{\sigma}(2) & -\mathfrak{b}_{\sigma}(2) & 0 \\
0 & -\mathfrak{b}_{\sigma}(2) & \mathfrak{a}_{\sigma}(3) & \ddots \\
0 & 0 & \ddots & \ddots
\end{array}\right)
$$

Такие матрицы могут иметь не более конечного числа собственных значений. Это доказывается тем же способом, что и лемма 2.1.1 в [1]. Доказательство основано на методе разбиения Глазмана, см. [3].

Используя эту лемму, а также соотношение (42), получаем

Следствие. Множество $\Theta$ простых полюсов матрии-фуункиий $U(k, \theta)$ конечно. Кроме того, при всех $k=0,1, \ldots$ функиии $U(k, \theta)$ ограниченъ в достаточно узком кольие $1-\epsilon \leqslant|\theta| \leqslant 1$.

Лемма 4.3. Для всех $\theta_{l} \in \Theta$ справедливы следующие утвержсения:

1. Существует независимая от $k$ матрица $\mathrm{m}\left(\theta_{l}\right)=\left(m\left(\sigma, \nu ; \theta_{l}\right)\right)_{\sigma, \nu \in \mathscr{C}}$, такая, что

$$
\operatorname{Res} U\left(k ; \theta_{l}\right)=E\left(k, \theta_{l}\right) \mathrm{m}\left(\theta_{l}\right), \quad k=0,1, \ldots
$$

2. Ее диагональные элементы $m\left(\nu, \nu ; \theta_{l}\right)$ удовлетворяют соотношению

$$
-\theta_{l} m\left(\nu, \nu, \theta_{l}\right)=\left\|\psi_{\nu}\right\|^{2},
$$

где функиия $\psi_{\nu}=\psi_{\nu}\left(\alpha, \theta_{l}\right)$ является решением уравнения $(3)$ с $\lambda=\lambda\left(\theta_{l}\right)$ и на каналах принимает значения

$$
\psi_{\nu}\left(\sigma(k), \theta_{l}\right)=e_{\sigma}\left(k, \theta_{l}\right) m\left(\sigma, \nu ; \theta_{l}\right), \quad \sigma, \nu \in \mathscr{C}, k \geqslant 0 .
$$

Доказательство. Рассмотрим окрестность $V$ точки $\theta_{l}$, такую, что $V^{\prime}:=$ $V \backslash\left\{\theta_{l}\right\}$ не содержит точек из $\mathscr{O}$. Зафиксируем $\nu \in \mathscr{C}$ и подставим в (38) вектор $\mathbf{n}=\left(\theta_{l}-\theta_{l}^{-1}\right)\left(\theta-\theta^{-1}\right)^{-1}\left(\theta-\theta_{l}\right) \boldsymbol{\delta}_{\nu}$, где $\boldsymbol{\delta}_{\nu}:=\delta(\sigma, \nu) \in l^{2}(\mathscr{C}), \delta(\sigma, \nu)-$ функция Кронекера. Мы получим функцию

$$
\psi_{\nu}(\alpha, \theta)= \begin{cases}\left(\left(\theta_{l}-\theta_{l}^{-1}\right)\left(\theta-\theta^{-1}\right)^{-1}\left(\theta-\theta_{l}\right) U(k, \theta) \boldsymbol{\delta}_{\nu}\right)(\alpha), & \alpha=\sigma(k) \in \mathscr{A}_{0} \cup \Gamma, \\ R_{1}(\theta) B(0) \boldsymbol{\psi}_{\nu}(1, \theta)(\alpha), & \alpha \in \mathscr{A}_{1} .\end{cases}
$$


В проколотой окрестности $V^{\prime}$ эта функция голоморфна и является решением уравнения (3) с $\lambda=\lambda(\theta)$. Если при каждом $\alpha \in \mathscr{A}$ существует предел

$$
\psi_{\nu}\left(\alpha, \theta_{l}\right)=\lim _{\theta \rightarrow \theta_{l}} \psi_{\nu}(\alpha, \theta),
$$

то $\psi_{\nu}(\alpha, \theta)$ голоморфна всюду в $V$ и (просто по непрерывности) при всех $\theta \in V$ является решением уравнения (3) с $\lambda=\lambda(\theta)$.

В случае когда $\theta_{l} \notin \mathscr{O}$ (т.е. $\theta_{l}$ не является полюсом матрицы-функции $\left.R_{1}\right)$, предел (47) существует при всех $\alpha \in \mathscr{A}$, поскольку матрица-функция $U(k, \theta)$ имеет в точке $\theta=\theta_{l}$ не более чем простой полюс.

Пусть теперь $\theta_{l} \in \mathscr{O}$, т. е. $\lambda\left(\theta_{l}\right)=\lambda_{s_{0}}$ для некоторого $s_{0}$. При $\alpha \in \mathscr{A}_{0} \cup \Gamma$ предел (47) все равно существует:

$$
\psi_{\nu}\left(\sigma(k) ; \theta_{l}\right)=\left(\operatorname{Res} U\left(k, \theta_{l}\right) \boldsymbol{\delta}_{\nu}\right)(\sigma) .
$$

Подставим во второе из уравнений (38) значение $\alpha=\sigma(0)$ (при этом значении $\alpha$ предел существует), умножим обе части на $\lambda_{s_{0}}-\lambda(\theta)$ и устремим $\theta$ к $\theta_{l}$. Мы получим

$$
0=\sum_{\lambda_{s}=\lambda_{s_{0}}} \sum_{\gamma \in \mathscr{C}} p\left(\lambda_{s}, \sigma(0)\right) p\left(\lambda_{s}, \gamma(0)\right) \mathfrak{b}_{\gamma}(0) \psi_{\nu}\left(\gamma(1) ; \theta_{l}\right)
$$

Поэтому

$$
\begin{aligned}
0 & =\sum_{\lambda_{s}=\lambda_{s_{0}}} \sum_{\sigma \in \mathscr{C}} \sum_{\gamma \in \mathscr{C}} \psi_{\nu}\left(\gamma(1) ; \theta_{l}\right) \mathfrak{b}_{\gamma}(0) p\left(\lambda_{s}, \gamma(0)\right) p\left(\lambda_{s}, \sigma(0)\right) \mathfrak{b}_{\sigma}(0) \psi_{\nu}\left(\sigma(1) ; \theta_{l}\right) \\
& =\sum_{\lambda_{s}=\lambda_{s_{0}}}\left(\sum_{\sigma \in \mathscr{C}} p\left(\lambda_{s}, \sigma(0)\right) \mathfrak{b}_{\sigma}(0) \psi_{\nu}\left(\sigma(1) ; \theta_{l}\right)\right)^{2},
\end{aligned}
$$

и (так как все члены этого соотношения вещественны)

$$
\sum_{\sigma \in \mathscr{C}} p\left(\lambda_{s}, \sigma(0)\right) \mathfrak{b}_{\sigma}(0) \psi_{\nu}\left(\sigma(1) ; \theta_{l}\right)=0 \quad \text { для каждого } s, \text { такого, что } \lambda_{s}=\lambda_{s_{0}} \text {. }
$$

Теперь мы можем перейти к пределу в правой части равенства (38) при всех $\alpha \in \mathscr{A}_{1}$. Поэтому при всех $\theta \in V$ для функции $\psi_{\nu}(\alpha, \theta)$ выполнено утверждение леммы 2.1.

Из соотношений (41) и (46) следует, что

$$
\psi_{\nu}(\sigma(k) ; \theta)=p_{\sigma}(k, \theta) a_{1}(\theta) \delta(\sigma, \nu)-e_{\sigma}(k, \theta) a_{2}(\theta)
$$

где

$$
a_{1}(\theta)=\frac{\left(\theta_{l}-\theta_{l}^{-1}\right)\left(\theta-\theta_{l}\right)}{\mathfrak{b}_{\nu}(0) e_{\nu}(1, \theta)}, \quad a_{2}(\theta)=\tau(\sigma, \nu ; \theta) a_{1}(\theta) ;
$$

здесь через $\tau(\sigma, \nu ; \theta)$ обозначены элементы обратной матрицы $T(\theta)^{-1}$.

Так как $\theta_{l}$ является простым полюсом матрицы-функции $U(k, \theta)$, то функции $a_{1}$ и $a_{2}$ голоморфны в $V$ и

$$
\psi_{\nu}\left(\sigma(k) ; \theta_{l}\right)=p_{\sigma}\left(k, \theta_{l}\right) a_{1}\left(\theta_{l}\right) \delta(\sigma, \nu)-e_{\sigma}\left(k, \theta_{l}\right) a_{2}\left(\theta_{l}\right) .
$$

Мы имеем

$$
a_{1}\left(\theta_{l}\right) \delta(\sigma, \nu)=\chi_{l}(\sigma) \delta(\sigma, \nu) \frac{e_{\sigma}\left(0, \theta_{l}\right)\left(\theta_{l}-\theta_{l}^{-1}\right)}{\mathfrak{b}_{\sigma}(0)\left\{e_{\sigma}\left(\cdot, \theta_{l}\right), \dot{e}_{\sigma}\left(\cdot, \theta_{l}\right)\right\}(0)},
$$


где

$$
\chi_{l}(\sigma)= \begin{cases}1, & e_{\sigma}\left(1, \theta_{l}\right)=0 \\ 0, & e_{\sigma}\left(1, \theta_{l}\right) \neq 0\end{cases}
$$

Кроме того,

$$
p_{\sigma}\left(k, \theta_{l}\right) e_{\sigma}\left(0, \theta_{l}\right) \chi_{l}(\sigma)=e_{\sigma}\left(k, \theta_{l}\right) \chi_{l}(\sigma),
$$

так как обе части этого соотношения удовлетворяют одному и тому же конечноразностному уравнению второго порядка и принимают одинаковые значения при $k=0,1$. Поэтому из соотношений (50) и (51) вытекает равенство

$$
\psi_{\nu}\left(\sigma(k), \theta_{l}\right)=e_{\sigma}\left(k, \theta_{l}\right) m\left(\sigma, \nu ; \theta_{l}\right),
$$

где

$$
m\left(\sigma, \nu ; \theta_{l}\right)=\chi_{l}(\sigma) \delta(\sigma, \nu) \frac{\theta_{l}-\theta_{l}^{-1}}{\mathfrak{b}_{\sigma}(0)\left\{e_{\sigma}\left(\cdot ; \theta_{l}\right), \dot{e}_{\sigma}\left(\cdot ; \theta_{l}\right)\right\}(0)}-a_{2}\left(\theta_{l}\right) .
$$

Соотношение (43) следует теперь из (45). Нам остается установить равенство (44).

Положим для краткости $\psi_{\nu}=\psi_{\nu}\left(\sigma(k) ; \theta_{l}\right)$. Согласно лемме 2.1 ,

$$
\sum_{\alpha \in \mathscr{A}_{1}} \psi_{\nu}\left(\alpha, \theta_{l}\right)^{2}=-\left(\theta_{l}^{-2}-1\right) \sum_{\sigma \in \mathscr{C}} \mathfrak{b}_{\sigma}(0)\left\{\psi_{\nu}, \dot{\psi}_{\nu}\right\}(0) .
$$

Из равенств (48), (53) вытекает, что

$$
\begin{aligned}
\mathfrak{b}_{\sigma}(0) & \left\{\psi_{\nu}, \dot{\psi}_{\nu}\right\}(0) \\
& =-\mathfrak{b}_{\sigma}(0)\left(\left\{e_{\sigma}\left(\cdot, \theta_{l}\right), \dot{e}_{\sigma}\left(\cdot, \theta_{l}\right)\right\}(0) a_{2}\left(\theta_{l}\right)+e_{\sigma}\left(1, \theta_{l}\right) \dot{a}_{1}\left(\theta_{l}\right) \delta(\sigma, \nu)\right) m\left(\sigma, \nu ; \theta_{l}\right) .
\end{aligned}
$$

Согласно (49), (52),

$$
\mathfrak{b}_{\sigma}(0) e_{\sigma}\left(1, \theta_{l}\right) \dot{a}_{1}\left(\theta_{l}\right)=\mathfrak{b}_{\sigma}(0) e_{\sigma}\left(1, \theta_{l}\right) \dot{a}_{1}\left(\theta_{l}\right)\left(1-\chi_{l}(\sigma)\right)=\left(\theta_{l}-\theta_{l}^{-1}\right)\left(1-\chi_{l}(\sigma)\right) .
$$

Используя соотношение (53), мы можем теперь привести (55) к виду

$$
\begin{aligned}
\mathfrak{b}_{\sigma}(0) & \left\{\psi_{\nu}, \dot{\psi}_{\nu}\right\}(0) \\
& =\mathfrak{b}_{\sigma}(0)\left\{e_{\sigma}\left(\cdot ; \theta_{l}\right), \dot{e}_{\sigma}\left(\cdot ; \theta_{l}\right)\right\}(0) m\left(\sigma, \nu ; \theta_{l}\right)^{2}-\left(\theta_{l}-\theta_{l}^{-1}\right) m\left(\sigma, \nu: \theta_{l}\right) \delta(\sigma, \nu) .
\end{aligned}
$$

Соответственно соотношение (54) принимает вид

$$
\begin{aligned}
\sum_{\alpha \in \mathscr{A}_{1}} \psi_{\nu}\left(\alpha, \theta_{l}\right)^{2} & =-\sum_{\sigma \in \mathscr{C}} \sum_{k=1}^{\infty}\left(e_{\sigma}\left(k, \theta_{l}\right) m\left(\sigma, \nu, \theta_{l}\right)\right)^{2}-\theta_{l} m\left(\nu, \nu ; \theta_{l}\right) \\
& =-\sum_{\alpha \in \mathscr{A}_{0}} \psi_{\nu}\left(\alpha, \theta_{l}\right)^{2}-\theta_{l} m(\nu, \nu),
\end{aligned}
$$

и, окончательно,

$$
-\theta_{l} m\left(\nu, \nu, \theta_{l}\right)=\sum_{\alpha \in \mathscr{A}} \psi_{\nu}\left(\alpha, \theta_{l}\right)^{2} .
$$

Замечание. Из соотношения (44) следует, что если при некотором $\nu$ справедливо равенство $m\left(\nu, \nu, \theta_{l}\right)=0$, то $\psi_{\nu}\left(\alpha, \theta_{l}\right) \equiv 0$ и, в частности, $m\left(\sigma, \nu, \theta_{l}\right)=0$ при всех $\sigma \in \mathscr{C}$. В случае когда $m\left(\nu, \nu ; \theta_{l}\right) \neq 0$, функция $\psi_{\nu}\left(\alpha ; \theta_{l}\right)$ является собственной функцией оператора $L$. Из положительности оператора $L$ следует, что $\theta_{l} \in(-1,0)$. 


\section{§5. Уравнения обратной задачи рассеяния}

Из равенства (15) следует, что ряд Фурье функции $E(k, \theta)$ имеет вид

$$
E(k, \theta)=C(k) \sum_{m=-\infty}^{\infty} A(k, m) \theta^{m}, \quad \theta \in \mathbb{T} ;
$$

здесь $A(k, m), C(k)$ определены соотношениями (27) и, кроме того, мы считаем, что $A(k, m)=0$ при $m<k$. Матрица рассеяния $S(\theta)$ является унитарной при $\theta \in \mathbb{T} \backslash \mathscr{O}$, и ее ряд Фурье имеет вид

$$
S(\theta)=\sum_{m=-\infty}^{\infty} \tilde{S}(m) \theta^{-m}, \quad \tilde{S}(m)=\frac{1}{2 i \pi} \int_{\mathbb{T}} S(\theta) \theta^{m-1} d \theta .
$$

Отсюда получается ряд Фурье функции $U(k, \theta)$ :

$$
\begin{aligned}
U(k, \theta) & =E\left(k, \theta^{-1}\right)+E(k, \theta) S(\theta) \\
& =C(k) \sum_{m=-\infty}^{\infty}\left\{A(k, m)+\sum_{l=-\infty}^{\infty} A(k, l) \tilde{S}(l+m)\right\} \theta^{-m} .
\end{aligned}
$$

Поэтому

$$
C(k)\left\{A(k, m)+\sum_{l=-\infty}^{\infty} A(k, l) \tilde{S}(l+m)\right\}=\frac{1}{2 \pi i} \int_{\mathbb{T}} U(k, \theta) \theta^{m-1} d \theta .
$$

Интеграл в правой части этого равенства равен сумме вычетов функции $\theta^{m-1} U(k, \theta)$ по всем ее полюсам, принадлежащим $\mathbb{D}$. Из леммы 4.1 следует, что при $m \geqslant k+1$ эта функция не имеет полюса в нуле и, согласно лемме 4.3 ,

$$
\frac{1}{2 \pi i} \int_{\mathbb{T}} U(k, \theta) \theta^{m-1} d \theta=\sum_{\theta_{l} \in \Theta} \theta_{l}^{m-1} \operatorname{Res} U\left(k, \theta_{l}\right)=\sum_{\theta_{l} \in \Theta} \theta_{l}^{m-1} E\left(k, \theta_{l}\right) \mathrm{m}\left(\theta_{l}\right) .
$$

Учитывая представление (57), мы получаем, что при $m \geqslant k+1$

$$
\begin{aligned}
\frac{1}{2 \pi i} \int_{\mathbb{T}} U(k, \theta) \theta^{m-1} d \theta & =C(k) \sum_{s=k}^{\infty} A(k, s) \sum_{\theta_{l} \in \Theta} \theta_{l}^{m+s-1} \mathrm{~m}\left(\theta_{l}\right) \\
& =C(k) \sum_{s=k}^{\infty} A(k, s) M(m+s),
\end{aligned}
$$

где

$$
M(n):=\sum_{\theta_{l} \in \Theta} \theta_{l}^{n}\left(\theta_{l}^{-1} \mathrm{~m}\left(\theta_{l}\right)\right) .
$$

Равенство (59) принимает теперь вид

$$
A(k, m)+\sum_{s=k}^{\infty} A(k, s) F(s+m)=0, \quad m=k+1, k+2, \ldots ;
$$

здесь

$$
F(n)=\tilde{S}(n)-M(n)=\frac{1}{2 i \pi} \int_{\mathbb{T}} S(\theta) \theta^{n-1} d \theta-\sum_{\theta_{l} \in \Theta} \theta_{l}^{n}\left\{\theta_{l}^{-1} \mathrm{~m}\left(\theta_{l}\right)\right\}
$$


Tак как $A(k, k)=I$, соотношение (60) может быть записано в виде

$$
F(k+m)+A(k, m)+\sum_{s=k+1}^{\infty} A(k, s) F(s+m)=0, \quad m \geqslant k+1 .
$$

Для каждого фиксированного $k$ это система уравнений относительно функций $\{A(k, m)\}_{m \geqslant k+1}$. Уравнения (62) называются основными уравнениями обратной задачи рассеяния.

Матрицы $A(k, m)$ являются диагональными, и их элементы $a_{\sigma}(k, m)$ зависят только от $\mathfrak{a}_{\sigma}(j), \mathfrak{b}_{\sigma}(j)$ при $j \geqslant k+1$. Мы утверждаем, что матрицы $F(n)$ обладают этими же свойствами.

Лемма 5.1. При всех $n \geqslant 1$ матрици $F(n)$ являются диагональнъми, $и$ их диагональные элементы $f_{\sigma}(n):=F(\sigma, \sigma ; n)$ зависят только от $a_{\sigma}(k, m)$, $m>k \geqslant\left[\frac{n-1}{2}\right]$.

Доказательство. Для всех $k$ и $j>k$ рассмотрим матрицы

$$
\begin{gathered}
\mathscr{X}(j)=\left(\begin{array}{c}
F(2 j+1) \\
F(2 j+2)
\end{array}\right), \quad B(k)=\left(\begin{array}{c}
A(k, k+1) \\
A(k, k+2)
\end{array}\right), \\
W(k)=\left(\begin{array}{ccc}
0 & -A(k, k+1) \\
0 & 0
\end{array}\right), \quad V(k, j)=\left(\begin{array}{cc}
A(k, 2 j-k) & A(k, 2 j-k+1) \\
A(k, 2 j-k-1) & A(k, 2 j-k)
\end{array}\right) .
\end{gathered}
$$

Элементы матриц $B, V$ и $W$ сами являются диагональными матрицами. Соотношение (62) может быть представлено в виде

$$
(I-W(k)) \mathscr{X}(k)=-\sum_{j=k+1}^{\infty} V(k, j) \mathscr{X}(j)-B(k) .
$$

Это дискретный аналог уравнения Вольтерра. В пространстве ограниченных матриц-функций оно имеет единственное решение, которое может быть получено с помощью стандартной процедуры:

$$
\begin{gathered}
\mathscr{X}(k)=\sum_{n=0}^{\infty} \mathscr{X}_{n}(k), \quad \mathscr{X}_{0}(k)=-(I-W(k))^{-1} B(k), \\
\mathscr{X}_{n}(k)=-\sum_{j>k}(I-W(k))^{-1} V(k, j) \mathscr{X}_{n-1}(j) .
\end{gathered}
$$

Используя соотношение (16), можно доказать, что эти ряды сходятся абсолютно и равномерно относительно $k$. Поэтому при каждом $\nu \in \mathscr{C}$ матрицы $F(k), k=$ $1,2, \ldots$, также являются диагональными, а их элементы $F(\nu, \nu ; k)$ однозначно определяются по $a_{\sigma}(j, m), m>j \geqslant\left[\frac{k-1}{2}\right]$, т. е. по значениям $\mathfrak{b}_{\nu}(j), \mathfrak{a}_{\nu}(j), j \geqslant$ $\left[\frac{k-1}{2}\right]+1$.

Теорема 2. Для систем частии, удовлетворяющих условиям A) и B), справедливы следуюшие утверждения:

1) уравнения (62) распадаются в систему независимых скалярных уравнений

$$
f_{\nu}(k+m)+a_{\nu}(k, m)+\sum_{s=k+1}^{\infty} a_{\nu}(k, s) f_{\nu}(s+m)=0, \quad m \geqslant k+1 \geqslant 1
$$


где

$$
f_{\nu}(n)=F(\nu, \nu ; n)=\frac{1}{2 \pi i} \int_{\mathbb{T}} s(\nu, \nu, \theta) \theta^{n-1} d \theta+\sum_{\theta_{l} \in \Theta} \theta_{l}^{n}\left(-\theta_{l}^{-1} m\left(\nu, \nu ; \theta_{l}\right)\right) ;
$$

2) для каждого $k \geqslant 0$ уравнения (63) имеют единственное решение $a_{\sigma}(k, m)$.

Доказательство. Первое утверждение является прямым следствием соотношения (61) и леммы 5.1. Согласно этой же лемме, функции $f_{\nu}(n), n \geqslant 1$, однозначно определяются по коэффициентам $\mathfrak{b}_{\nu}(k), \mathfrak{a}_{\nu}(k), k \geqslant 1$. Таким образом, соотношение (63) совпадает с основным уравнением обратной задачи рассеяния для системы $(6)$ с граничным условием $\xi(\nu(0))=0$. Однозначная разрешимость этого уравнения хорошо известна (см., например, [9]).

\section{§6. Восстановление функций $f_{\nu}(n)$ по наблюдениям на бесконечности}

Согласно теореме 2 , функции $f_{\nu}(n), n \geqslant 1$, участвующие в основном уравнении обратной задачи, определяются по диагональным элементам $s(\nu, \nu, \theta)$ матрицы рассеяния, а также по диагональным элементам матриц $\mathrm{m}\left(\theta_{l}\right)$.

Матрица рассеяния определяется по наблюдениям на бесконечно удаленных участках каналов; поэтому элементы $s(\nu, \nu, \theta)$ известны. В этом разделе мы восстанавливаем значения $m\left(\nu, \nu, \theta_{l}\right)$.

Рассмотрим подпространство $\Lambda \subset l^{2}(\mathscr{A})$, порожденное всеми собственными функциями оператора $L$, и представим его в виде

$$
\Lambda=\Omega \oplus \Omega^{\perp},
$$

где $\Omega$ обозначает подпространство, порожденное собственными функциями $\omega(\alpha)$, обращающимися в нуль на $\Gamma \cup \mathscr{A}_{0}$, т.е. такими, что $\omega(\sigma(k))=0, k=$ $0,1,2, \ldots, \sigma \in \mathscr{C}$.

Это подпространство, вообе говоря, отлично от нулевого. Колебания, отвечающие собственным функциям из $\Omega$, не оказывают влияния на каналы. С другой стороны, легко видеть, что возмущения каналов не вызывают колебаний из $\Omega$.

Поэтому все колебания, наблюдаемые на бесконечности, принадлежат $\Omega^{\perp}$. Через $\omega_{q}=\left(2-\theta_{q}-\theta_{q}^{-1}\right)^{1 / 2}, \theta_{q} \in(-1,0)$, мы обозначаем соответствующие частоты. Таким образом, множество $\Theta^{\perp}=\left\{\theta_{q}\right\}$ также известно.

Собственные функции $\psi_{\nu}\left(\alpha, \theta_{l}\right)$, построенные в лемме 4.3 , принадлежат $\Omega^{\perp}$. В самом деле, очевидно, что $\left\langle\psi_{\nu}, \omega\right\rangle=0$, если собственные функции $\omega \in \Omega$ и $\psi_{\nu}$ отвечают разным собственным значениям. Если же собственные значения совпадают, воспользуемся леммой 2.1:

$$
\left\langle\psi_{\nu}, \omega\right\rangle=\sum_{\alpha \in \mathscr{A}_{1}} \psi_{\nu}\left(\alpha, \theta_{l}\right) \overline{\omega(\alpha)}=\left(\theta_{l}^{-2}-1\right)^{-1} \sum_{\sigma \in \mathscr{C}} \mathfrak{b}_{\sigma}(0)\left\{\dot{\psi}\left(\sigma(\cdot), \theta_{l}\right), \overline{\omega(\sigma(\cdot))}\right\}(0)=0
$$

и, таким образом, $\psi_{\nu}\left(\alpha, \theta_{l}\right) \in \Omega^{\perp}$.

Очевидно, что $\Theta \subset \Theta^{\perp}$. Равенство, вообще говоря, может не иметь места, потому что мы не можем утверждать, что все функции из $\Omega^{\perp}$ являются функциями вида, описанного в лемме 4.3. Так как только точки из $\Theta$ участвуют в конструкции функций $f_{\nu}$, нам нужно определить это множество по данным наблюдений на бесконечности. 
Лемма 6.1. Пусть $\sigma, \nu \in \mathscr{C}, \sigma \neq \nu, u Q(\theta)=\prod_{\theta_{q} \in \Theta^{\perp}}\left(\theta-\theta_{q}\right)$. Тогда

$$
\frac{1}{2 \pi i} \int_{\mathbb{T}} \frac{s(\sigma, \nu, \theta) Q(\theta)}{\left(\theta-\theta_{q}\right) Q^{\prime}\left(\theta_{q}\right)} d \theta= \begin{cases}m\left(\sigma, \nu, \theta_{l}\right), & \theta_{q}=\theta_{l} \in \Theta \\ 0, & \theta_{q} \notin \Theta .\end{cases}
$$

Доказательство. Из соотношения (61) и леммы 5.1 следует, что

$$
\begin{aligned}
0 & =\frac{1}{2 \pi i} \int_{\mathbb{T}} s(\sigma, \nu ; \theta) \theta^{n-1} d \theta-\sum_{\theta_{l} \in \Theta} \theta_{l}^{n-1} m\left(\sigma, \nu ; \theta_{l}\right) \\
& =\frac{1}{2 \pi i} \int_{\mathbb{T}}\left\{s(\sigma, \nu ; \theta)-\sum_{\theta_{l} \in \Theta}\left(\theta-\theta_{l}\right)^{-1} m\left(\sigma, \nu ; \theta_{l}\right)\right\} \theta^{n-1} d \theta, \quad n=1,2, \ldots,
\end{aligned}
$$

и так как $Q(\theta)\left[\left(\theta-\theta_{q}\right) Q^{\prime}\left(\theta_{q}\right)\right]^{-1}$ является полиномом, то

$$
\frac{1}{2 \pi i} \int_{\mathbb{T}}\left\{s(\sigma, \nu ; \theta)-\sum_{\theta_{l} \in \Theta}\left(\theta-\theta_{l}\right)^{-1} m\left(\sigma, \nu ; \theta_{l}\right)\right\} \frac{Q(\theta)}{\left(\theta-\theta_{q}\right) Q^{\prime}\left(\theta_{q}\right)} d \theta=0 .
$$

Равенство (65) получается теперь с помощью теоремы о вычетах.

Следствие 1. Все полюсъ $\theta_{l} \in \Theta$, при которых матрииа $\mathrm{m}\left(\theta_{l}\right)$ не является диагональной, а также все недиагональные элементы $\mathrm{m}\left(\sigma, \nu ; \theta_{l}\right)$ этой матриць могут быть определены по матрице рассеяния $S(\theta), \theta \in \mathbb{T}, u$ мноэсеству $\Theta^{\perp}$.

Следствие 2. Матрицъ $\mathrm{m}\left(\theta_{l}\right)$ являются эрмитовыми.

В самом деле, так как матрица $S$ симметрична, то в силу соотношения (65) матрица $\mathrm{m}\left(\theta_{l}\right)$ также является симметричной. Осталось воспользоваться вещественностью матрицы $\mathrm{m}\left(\theta_{l}\right)$.

Для каждого $\theta_{q} \in \Theta^{\perp}$ рассмотрим собственное подпространство $\Lambda\left(\theta_{q}\right)$ оператора $L$, отвечающее собственному значению $\lambda\left(\theta_{q}\right)=2-\theta_{q}-\theta_{q}^{-1}$. Как и ранее, обозначим через $\Omega\left(\theta_{q}\right)$ множество собственных функций, обращающихся в нуль на $\mathscr{A}_{0} \cup \Gamma$, и положим $\Omega^{\perp}\left(\theta_{q}\right)=\Lambda\left(\theta_{q}\right) \ominus \Omega\left(\theta_{q}\right)$.

Для каждой собственной функции $\phi(\alpha) \in \Lambda\left(\theta_{q}\right)$ определим ее индикатор $\phi(\infty)=\phi(\infty, \sigma) \in l^{2}(\mathscr{C})$ равенством $\phi(\infty, \sigma)=\lim _{k \rightarrow \infty} \theta_{q}^{-k} \phi(\sigma(k))$. Имеем

$$
\phi(\alpha)=E\left(k, \theta_{q}\right) \phi(\infty), \quad \alpha=\sigma(k), \sigma \in \mathscr{C}, k=0,1,2, \ldots
$$

Индикатор функции ф определяет ее асимптотическое поведение и, следовательно, может быть получен из наблюдений на бесконечности. Он показывает, как распределяется энергия между различными каналами, и в этом смысле для дискретного спектра является аналогом матрицы рассеяния.

Индикаторы функций, принадлежащих $\Lambda\left(\theta_{q}\right)$, образуют линейное подпространство в $l^{2}(\mathscr{C})$, и мы обозначим его через $\left[\Lambda\left(\theta_{q}\right)\right]$. Так как $\phi(\infty)=0$ при $\phi \in \Omega\left(\theta_{q}\right)$, то $\left[\Lambda\left(\theta_{q}\right)\right]=\left[\Omega^{\perp}\left(\theta_{q}\right)\right]$. Таким образом, каждая функция из $\Omega^{\perp}\left(\theta_{q}\right)$ однозначно определяется своим индикатором. Соответственно каждая функция $\phi \in \Omega^{\perp}$ может быть однозначно определена по своему индикатору и собственному значению, т. е. по паре $\left\{\theta_{q}, \phi(\infty, \sigma)\right\}$.

Пусть $\mathscr{E}-$ множество всех корней определителя $\operatorname{det} E(1, \theta)=\prod_{\sigma \in \mathscr{C}} e_{\sigma}(1, \theta)$. 
Лемма 6.2. Пусть $\theta_{q} \in \Theta^{\perp} u \theta_{q} \notin \mathscr{O} \cup \mathscr{E}$. Тогда точка $\theta_{q}$ является полюсом матрии, $U(k, \theta)$, m.е. $\theta_{q} \in \Theta$. Кроме того,

$$
\operatorname{Ker} T\left(\theta_{q}\right)=\operatorname{Im} \mathrm{m}\left(\theta_{q}\right)=\left[\Lambda\left(\theta_{q}\right)\right]
$$

где $\operatorname{Ker} T\left(\theta_{q}\right)$ и $\operatorname{Im} \mathrm{m}\left(\theta_{q}\right)$ обозначают соответственно ядро матрицы $T\left(\theta_{q}\right)$ и образ матрицы $\mathrm{m}\left(\theta_{q}\right)$.

Доказательство. С учетом того, что $\theta_{q} \notin \mathscr{O}$, из теоремы 1 вытекает, что функция $\phi(\alpha)$ принадлежит $\Lambda\left(\theta_{q}\right)$ тогда и только тогда, когда она удовлетворяет уравнению (66) и граничному условию (10).

Поэтому

$$
\left.\left(E\left(0, \theta_{q}\right)-R\left(\theta_{q}\right) B(0) E\left(1, \theta_{q}\right)\right)\right) \phi(\infty)=T\left(\theta_{q}\right) \phi(\infty)=0,
$$

т. е. $\phi(\infty) \in \operatorname{Ker} T\left(\theta_{q}\right)$ и $\theta_{q}$ является полюсом функции $T(\theta)^{-1}$. В частности, отсюда следует, что $\theta_{q} \in \Theta$ и $\left[\Lambda\left(\theta_{q}\right)\right]=\operatorname{Ker} T\left(\theta_{q}\right)$.

Мы предположили, что $\theta_{q} \notin \mathscr{E}$. Поэтому разложения функций $T(\theta), T(\theta)^{-1}$ и $E(1, \theta)$ в окрестности точки $\theta_{q}$ имеют вид

$$
\begin{aligned}
T(\theta) & =T\left(\theta_{q}\right)+\dot{T}\left(\theta_{q}\right)\left(\theta-\theta_{q}\right)+\ldots, \\
T(\theta)^{-1} & =N_{-1}\left(\theta-\theta_{q}\right)^{-1}+N_{0}+N_{1}\left(\theta-\theta_{q}\right)+\ldots, \\
E(1, \theta)^{-1} & =E\left(1, \theta_{q}\right)^{-1}-E\left(1, \theta_{q}\right)^{-2} \dot{E}\left(1, \theta_{q}\right)\left(\theta-\theta_{q}\right)+\ldots
\end{aligned}
$$

Из соотношений (67) и (68) вытекает, что $\operatorname{Ker} T\left(\theta_{q}\right)=\operatorname{Im} N_{-1}$, а используя соотношения (68), (69) и (43), (41), мы получаем, что $\mathrm{m}\left(\theta_{q}\right)=-N_{-1} B(0)^{-1} E\left(1, \theta_{q}\right)^{-1}$. Поэтому $\left[\Lambda\left(\theta_{q}\right)\right]=\operatorname{Ker} T\left(\theta_{q}\right)=\operatorname{Im} N_{-1}=\operatorname{Im} \mathrm{m}\left(\theta_{q}\right)$.

Определение. Подпространство $\Lambda\left(\theta_{q}\right)$ называется резонансным, если $\theta_{q} \in$ $\mathscr{O} \cup \mathscr{E}$ и $\Lambda\left(\theta_{q}\right)$ содержит хотя бы одну функцию, которая обращается в нуль на всех каналах, кроме одного (иными словами, индикатор $\left[\Lambda\left(\theta_{q}\right)\right]$ содержит по меньшей мере одну базисную функцию $\boldsymbol{\delta}_{\nu}=\delta(\sigma, \nu), \sigma \in \mathscr{C}$, пространства $\left.l^{2}(\mathscr{C})\right)$.

В дальнейшем мы предполагаем, что резонансных подпространств не существует. В этом случае мы находим все полюсы матриц-функций $U(k, \theta)$, а также значения $\nu \in \mathscr{C}$, при которых диагональные элементы $m\left(\nu, \nu, \theta_{l}\right)$ соответствующих матриц $\mathrm{m}\left(\theta_{l}\right)$ не обращаются в нуль.

Положим

$\Theta_{1}=\left\{\theta_{l} \in \Theta: \mathrm{m}\left(\theta_{l}\right)\right.$ не является диагональной матрицей $\}, \quad \Theta_{2}=\Theta \backslash \Theta_{1}$, $\Theta_{1}^{\perp}=\left\{\theta_{q} \in \Theta^{\perp}:\left[\Lambda\left(\theta_{q}\right)\right]\right.$ не содержит базисных векторов $\left.\delta_{\nu}\right\}, \quad \Theta_{2}^{\perp}=\Theta^{\perp} \backslash \Theta_{1}^{\perp}$.

Множества $\Theta^{\perp}, \Theta_{1}^{\perp}$ и $\Theta_{2}^{\perp}$ могут быть найдены из наблюдений на бесконечности, а множество $\Theta_{1}$ находится применением леммы 6.1. Остается определить множество $\Theta_{2}$.

Очевидно, что

$$
\Theta_{2} \cap \Theta_{1}^{\perp}=\varnothing
$$

и, кроме того,

$$
\Theta_{2}^{\perp} \cap(\mathscr{O} \cup \mathscr{E})=\varnothing
$$

так как в противном случае $\Lambda\left(\theta_{q}\right)$ являлось бы резонансным подпространством при некотором $\theta_{q} \in \Theta_{2}^{\perp} \cap(\mathscr{O} \cup \mathscr{E})$. 
Из леммы 6.2 вытекает, что $\Theta_{2}^{\perp} \subset \Theta$ и $\Theta=\left(\Theta \cap \Theta_{1}^{\perp}\right) \cup\left(\Theta \cap \Theta_{2}^{\perp}\right)=\left(\Theta_{1} \cap \Theta_{1}^{\perp}\right) \cup$ $\left(\Theta_{2} \cap \Theta_{1}^{\perp}\right) \cup \Theta_{2}^{\perp}=\left(\Theta_{1} \cap \Theta_{1}^{\perp}\right) \cup \Theta_{2}^{\perp}$ и, окончательно,

$$
\Theta=\left(\Theta_{1} \cap \Theta_{1}^{\perp}\right) \cup \Theta_{2}^{\perp} .
$$

Все множества в правой части этого выражения известны и, таким образом, множество $\Theta$ определено.

Теперь при каждом $\theta_{l} \in \Theta$ мы определяем множество

$$
N\left(\theta_{l}\right):=\left\{\nu \in \mathscr{C}: m\left(\nu, \nu ; \theta_{l}\right) \neq 0\right\} .
$$

Очевидно, что $N\left(\theta_{l}\right) \subset N_{1}\left(\theta_{l}\right) \cup N_{2}\left(\theta_{l}\right)$, где

$$
\begin{gathered}
N_{1}\left(\theta_{l}\right):=\left\{\nu \in N\left(\theta_{l}\right): m\left(\sigma, \nu, \theta_{l}\right) \neq 0 \text { для хотя бы одного } \sigma \neq \nu\right\}, \\
N_{2}\left(\theta_{l}\right):=\left\{\nu \in \mathscr{C}: \boldsymbol{\delta}_{\nu} \in\left[\Lambda\left(\theta_{l}\right)\right]\right\} .
\end{gathered}
$$

Множества $N_{1}\left(\theta_{l}\right)$ и $N_{2}\left(\theta_{l}\right)$ известны: $N_{1}\left(\theta_{l}\right)$ находится с помощью леммы 6.1 , a $N_{2}\left(\theta_{l}\right)$ может быть определено из наблюдений на бесконечности.

При $\theta_{l} \in \Theta_{1} \cap \Theta_{1}^{\perp}$ индикаторное множество $\left[\Lambda\left(\theta_{l}\right)\right]$ не содержит базисных векторов; поэтому $N_{2}\left(\theta_{l}\right)=\varnothing$ и

$$
N\left(\theta_{l}\right)=N_{1}\left(\theta_{l}\right) \cup N_{2}\left(\theta_{l}\right) .
$$

Пусть теперь $\theta_{l} \in \Theta_{2}^{\perp}$. Из соотношения (70) и леммы 6.2 вытекает, что $\left[\Lambda\left(\theta_{l}\right)\right]=\operatorname{Im} \mathrm{m}\left(\theta_{l}\right)$. Поэтому, если $\nu \in N_{2}\left(\theta_{l}\right) \backslash N_{1}\left(\theta_{l}\right)$, то $m\left(\sigma, \nu, \theta_{l}\right)=0$ для всех $\sigma \neq \nu$ и, кроме того, $\boldsymbol{\delta}_{\nu} \in\left[\Lambda\left(\theta_{l}\right)\right]=\operatorname{Im} \mathrm{m}\left(\theta_{l}\right)$. Другими словами, $\boldsymbol{\delta}_{\nu}=\mathrm{m}\left(\theta_{l}\right) \boldsymbol{f}$ при подходящем $\boldsymbol{f}=f(\gamma), \gamma \in \mathscr{C}$. В частности,

$$
1=m\left(\nu, \nu, \theta_{l}\right) f(\nu)+\sum_{\gamma \neq \nu} m\left(\nu, \gamma, \theta_{l}\right) f(\gamma)
$$

С учетом того, что матрица $\mathrm{m}\left(\theta_{l}\right)$ эрмитова, мы получаем, что $1=m\left(\nu, \nu, \theta_{l}\right) f(\nu)$, а следовательно, $m\left(\nu, \nu, \theta_{l}\right) \neq 0$, т. е.

$$
N_{2}\left(\theta_{l}\right) \backslash N_{1}\left(\theta_{l}\right) \subset N\left(\theta_{l}\right) \backslash N_{1}\left(\theta_{l}\right) .
$$

С другой стороны, если $\nu \in N\left(\theta_{l}\right) \backslash N_{1}\left(\theta_{l}\right)$, то $m\left(\nu, \nu, \theta_{l}\right) \neq 0$ и, кроме того, $m\left(\sigma, \nu, \theta_{l}\right)=0$ при всех $\sigma \neq \nu$. Согласно лемме 4.3, индикатор собственной функции $m\left(\nu, \nu, \theta_{l}\right)^{-1} \psi_{\nu}\left(\alpha, \theta_{l}\right)$ равен $\boldsymbol{\delta}_{\nu}$. Поэтому $\boldsymbol{\delta}_{\nu} \in\left[\Lambda\left(\theta_{l}\right)\right]$ и соответственно $N\left(\theta_{l}\right) \backslash N_{1}\left(\theta_{l}\right) \subset N_{2}\left(\theta_{l}\right) \backslash N_{1}\left(\theta_{l}\right)$. Отсюда, с учетом соотношения (73) и включения $N_{1}\left(\theta_{l}\right) \subset N\left(\theta_{l}\right)$, мы получаем $(72)$.

Таким образом, множества $\Theta$ и $N\left(\theta_{l}\right), \theta_{l} \in \Theta$, определяются соотношениями (72), (73) с помощью $\Theta_{1}, \Theta_{1}^{\perp}, \Theta_{2}^{\perp}$ и $N_{1}\left(\theta_{l}\right), N_{2}\left(\theta_{l}\right)$. Все эти множества могут быть найдены по наблюдениям на бесконечности.

Остается определить диагональные элементы матриц $\mathrm{m}\left(\theta_{l}\right)$ для $\theta_{l} \in \Theta$. Тогда функции $f_{\nu}(n)$, участвующие в основном уравнении обратной задачи рассеяния, могут быть определены из равенства (61).

Зафиксируем $\theta_{l} \in \Theta$ и $\nu \in N\left(\theta_{l}\right)$. Рассмотрим функцию $\psi_{\nu}=\psi_{\nu}\left(\alpha, \theta_{l}\right) \in$ $\Omega^{\perp}\left(\theta_{l}\right)$, построенную в лемме 4.3. Так как $\psi_{\nu}(\infty, \sigma)=m\left(\sigma, \nu, \theta_{l}\right)$, лемма 6.1 позволяет определить $m\left(\sigma, \nu, \theta_{l}\right)(1-\delta(\sigma, \nu))$ по матрице рассеяния. Возможны следующие случаи: 
1. Пусть $m\left(\sigma, \nu, \theta_{l}\right)(1-\delta(\sigma, \nu))=0$. Тогда подпространство $\Omega^{\perp}\left(\theta_{l}\right)$ содержит единственную функцию $\phi_{1}=\phi_{1}\left(\alpha, \theta_{l}\right)$, такую, что $\phi_{1}(\infty, \sigma)=\delta(\sigma, \nu)$. Поэтому $\psi_{\nu}\left(\alpha, \theta_{l}\right)=m\left(\nu, \nu, \theta_{l}\right) \phi_{1}\left(\alpha, \theta_{l}\right)$ и из соотношения (44) вытекает равенство

$$
\theta_{l}^{-1} m\left(\nu, \nu, \theta_{l}\right)=-\left\|\phi_{1}\right\|^{-2} .
$$

2. Если $m\left(\sigma, \nu, \theta_{l}\right)(1-\delta(\sigma, \nu)) \neq 0$ и подпространство $\Omega^{\perp}\left(\theta_{l}\right)$ содержит единственную функцию $\phi_{0}=\phi_{0}\left(\alpha, \theta_{l}\right)$, такую, что $\phi_{0}(\infty, \sigma)=m\left(\sigma, \nu, \theta_{l}\right)$ при $\sigma \neq \nu$, то $\psi_{\nu}=\phi_{0}, m\left(\nu, \nu, \theta_{l}\right)=\phi_{0}(\infty, \nu)$ и из соотношений (44) следует, что

$$
\theta_{l}^{-1} m\left(\nu, \nu, \theta_{l}\right)=-\theta_{l}^{-2}\left\|\phi_{0}\right\|^{2}=\theta_{l}^{-1} \phi_{0}(\infty, \nu) .
$$

3. Пусть, наконец, подпространство $\Omega^{\perp}\left(\theta_{l}\right)$ содержит две функции, индикаторы которых совпадают с $m\left(\sigma, \nu, \theta_{l}\right)$ при $\sigma \neq \nu$. Тогда найдутся единственные функции $\phi_{2}, \phi_{1} \in \Omega^{\perp}\left(\theta_{l}\right)$, такие, что $\phi_{1}(\infty, \sigma)=\delta(\sigma, \nu)$ и $\phi_{2}(\infty, \sigma)=$ $m\left(\sigma, \nu, \theta_{l}\right)(1-\delta(\nu, \sigma))$. Следовательно,

$$
\psi_{\nu}=\phi_{2}+x \phi_{1}
$$

где $x=m\left(\nu, \nu, \theta_{l}\right)$ - это значение, которое мы должны определить. Имеем

$$
\left\|\psi_{\nu}\right\|^{2}=\left\|\phi_{2}\right\|^{2}+2 x\left\langle\phi_{2}, \phi_{1}\right\rangle+x^{2}\left\|\phi_{1}\right\|^{2} \quad \text { и } \quad\left\langle\psi_{\nu}, \phi_{1}\right\rangle=\left\langle\phi_{2}, \phi_{1}\right\rangle+x\left\|\phi_{1}\right\|^{2} .
$$

С учетом соотношения $\left\|\psi_{\nu}\right\|^{2}=-\theta_{l} m\left(\nu, \nu, \theta_{l}\right)=-\theta_{l} x$ получаем

$$
-\theta_{l} x=\left\|\phi_{2}\right\|^{2}+2 x\left\langle\psi_{\nu}, \phi_{1}\right\rangle-x^{2}\left\|\phi_{1}\right\|^{2} .
$$

Так как $\phi_{1}\left(\sigma(k), \theta_{l}\right)=e_{\sigma}\left(k, \theta_{l}\right)$, из леммы 2.1 следует, что

$$
\left(\theta_{l}^{-2}-1\right) \sum_{\alpha \in \mathscr{A}_{1}} \psi_{\nu}\left(\alpha, \theta_{l}\right) \phi_{1}\left(\alpha, \theta_{l}\right)=\mathfrak{b}_{\nu}(0)\left\{\dot{\psi}_{\nu}\left(\nu(\cdot), \theta_{l}\right), e_{\nu}\left(\cdot, \theta_{l}\right)\right\}(0) .
$$

Здесь, согласно соотношениям (48), (49), (52) и (21),

$$
\begin{aligned}
\mathfrak{b}_{\nu} & (0)\left\{\dot{\psi}_{\nu}\left(\nu(\cdot), \theta_{l}\right), e_{\nu}\left(\cdot, \theta_{l}\right)\right\}(0) \\
& =\mathfrak{b}_{\nu}(0) \dot{a}_{1}\left(\theta_{l}\right) e_{\nu}\left(1, \theta_{l}\right)-\mathfrak{b}_{\nu}(0)\left\{\dot{e}_{\nu}\left(\cdot, \theta_{l}\right), e_{\nu}\left(\cdot, \theta_{l}\right)\right\}(0) a_{2}\left(\theta_{l}\right) \\
& =\left(\theta_{l}-\theta_{l}^{-1}\right)\left(1-\chi_{l}(\nu)\right)+\left(\theta_{l}-\theta_{l}^{-1}\right) \chi_{l}(\nu)+\mathfrak{b}_{\nu}(0)\left\{\dot{e}_{\nu}\left(\cdot, \theta_{l}\right), e_{\nu}\left(\cdot, \theta_{l}\right)\right\}(0) m\left(\nu, \nu, \theta_{l}\right) \\
& =\left(\theta_{l}-\theta_{l}^{-1}\right)-\left(\bar{\theta}_{l}^{-2}-1\right) \sum_{\alpha \in \mathscr{A}_{1}} \psi_{\nu}\left(\alpha, \theta_{l}\right) \phi_{1}\left(\alpha, \theta_{l}\right) .
\end{aligned}
$$

Поэтому

$$
\left\langle\psi_{\nu}, \phi_{1}\right\rangle=\sum_{\alpha \in \mathscr{A}} \psi_{\nu}\left(\alpha, \theta_{l}\right) \phi_{1}\left(\alpha, \theta_{l}\right)=-\theta_{l}
$$

и соотношение (74) принимает вид

$$
x^{2}+x \theta_{l}\left\|\phi_{1}\right\|^{-2}-\left\|\phi_{2}\right\|^{2}\left\|\phi_{1}\right\|^{-2}=0 .
$$

Окончательно, с учетом того, что $-\theta_{l} x=-\theta_{l} m\left(\nu, \nu, \theta_{l}\right)=\left\|\psi_{\nu}\right\|^{2}>0$, получаем

$$
\theta_{l}^{-1} m\left(\nu, \nu, \theta_{l}\right)=-\frac{1}{2}\left\|\phi_{1}\right\|^{-2}\left(1+\sqrt{1+4\left\|\phi_{1}\right\|^{2}\left\|\phi_{2}\right\|^{2} \theta_{l}^{-2}}\right) .
$$


Таким образом, значения $\theta_{l}^{-1} m\left(\nu, \nu, \theta_{l}\right)$ могут быть выражены через нормы соответствующих собственных функций $\phi_{j}\left(\theta_{l}\right)(j=0,1,2)$, индикаторы которых известны. Эти нормы выражаются через энергию $E_{j}=\omega\left(\theta_{j}\right)\left\|\phi_{j}\right\|^{2}$ соответствующих свободных колебаний, которая может быть определена из наблюдений на бесконечности.

Данными рассеяния мы называем матричу рассеяния $S(\theta), \theta \in \mathbb{T}$, собственные значения $\lambda\left(\theta_{l}\right)=2-\theta_{l}-\theta_{l}^{-1}$, а также индикаторы и нормы соответствующих собственных функиий. Эти данные могут быть получены из наблюдений на бесконечности.

Теорема 3. Предположим, что L не имеет резонансных подпространств. Тогда по данным рассеяния можно однозначно восстановить все элементы $L(\alpha, \beta)$ при $\alpha, \beta \in \mathscr{A}_{0}$, а также решения Йоста $e_{\sigma}(k, \theta), \sigma \in \mathscr{C}, k \geqslant 1$.

Доказательство. Приведенное выше построение позволяет определить простые полюсы матриц-функций $U(k, \theta)$, а также вычеты в этих полюсах. По ним, в свою очередь, определяются функции $f_{\nu}(n), n \geqslant 1$. Теперь, решая уравнение (63), можно найти $a_{\sigma}(k, m), \sigma \in \mathscr{C}, m \geqslant k \geqslant 0$, и (используя соотношения (18), $(19))$ коэффициенты $\mathfrak{a}_{\sigma}(k), \mathfrak{b}_{\sigma}(k)$ и, наконец, множители $c_{\sigma}(k)=\prod_{j \geqslant k} \mathfrak{b}_{\sigma}(j)^{-1}$ (для $k \geqslant 1$ ). Решения Йоста восстанавливаются теперь из соотношения (24).

Замечание. Значения $e_{\sigma}(0, \theta)$ могут быть получены из соотношения

$$
e_{\sigma}(0, \theta)=\mathfrak{b}_{\sigma}(0)^{-1}\left\{\left(\mathfrak{a}_{\sigma}(1)-2+\theta+\theta^{-1}\right) e_{\sigma}(1, \theta)-\mathfrak{b}_{\sigma}(1) e_{\sigma}(2, \theta)\right\}
$$

с точностью до (неизвестного) множителя $\mathfrak{b}_{\sigma}(0)^{-1}$.

\section{ЛитературА}

[1] 3. С. Агранович. В. А. Марченко, Обратная задача теории рассеяния, Изд-во Харьковского госуниверситета, 1960.

[2] R. Carlson, Inverse eigenvalue problems on directed graphs, Trans. Amer. Math. Soc., 351:10 (1999), 4069-4088.

[3] И. М. Глазман, Прямые методы качественного спектрального анализа сингулярных дифберенциальных операторов, Физматгиз, 1963.

[4] Н. И. Герасименко, Б. С. Павлов, Задача рассеяния на некомпактных графах, Теор. матем. физика, 74:3 (1988), 345-359.

[5] B. Gutkin, U. Smilansky, Can one hear the shape of a graph? J. Phys. A, 34:31 (2001), 6061-6068.

[6] V. Kostrykin, R. Schrader, The inverse scattering problem for metric graphs and the travelling salesman problem http://arxiv.org/math-ph/0603010.

[7] P. Kuchment, Quantum graphs. II. Some spectral properties of quantum and combinatorial graphs, J. Phys. A, 38:22 (2005), 4887-4900.

[8] P. Kurasov, M. Nowaczyk, Inverse spectral problem for quantum graphs, J. Phys. A, 38:22 (2005), 4901-4915.

[9] В. А. Марченко, Введение в теорию обратных задач спектрального анализа, Акта, Харьков, 2005.

Department of Matehmatical Sciences,

Norwegian University of Science and Technology

e-mail: yura@math.ntnu.no

Математическое отделение Института низких температур

Академии наук Украины, Харьков
Поступило в редакцию 27 ноября 2006 г. 